\title{
Longitudinal associations between formal volunteering and well-being among retired older people: Follow-up results from a randomized controlled trial
}

\author{
Michelle I Jongenelis ${ }^{\mathrm{a}^{*}}$, Ben Jackson $^{\mathrm{b}}$, Robert U Newton ${ }^{\mathrm{c}}$, and Simone Pettigrew ${ }^{\mathrm{de}}$

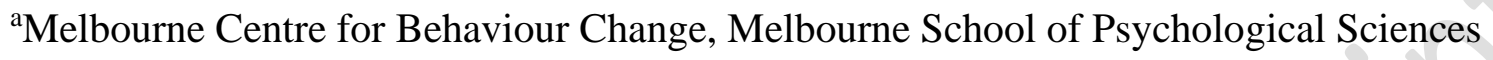 \\ University of Melbourne \\ P: +61 039035 4979; Michelle.jongenelis@unimelb.edu.au \\ ${ }^{\mathrm{b}}$ School of Human Sciences (Exercise and Sports Science), University of Western Australia \\ Ben.jackson@uwa.edu.au

 \\ r.newton@ecu.edu.au \\ ${ }^{\mathrm{d}}$ The George Institute for Global Health \\ SPettigrew@georgeinstitute.org.au
}

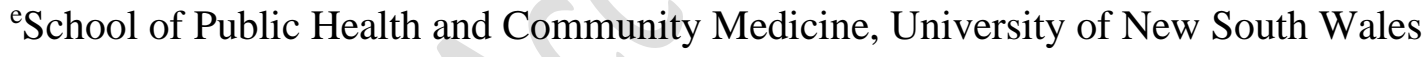

Funding statement: This study was funded by an Australian Research Council Discovery Grant (DP140100365).

Acknowledgements: The authors wish to thank Nicole Biagioni, Zenobia Talati, and the team of staff and students at Curtin University and the Vario Health Clinic at Edith Cowan University for their assistance with data collection. 


\title{
Longitudinal associations between formal volunteering and well-being among retired older people: Follow-up results from a randomized controlled trial
}

\begin{abstract}
Volunteering has been identified as a potential mechanism for improving the psychosocial health of older adults. Utilizing a randomized controlled trial approach, the present study assessed the extent to which commencing volunteering can improve psychosocial health outcomes for older people. Fully-retired Australian adults aged $60+$ years $(N=445)$ were assessed at baseline and allocated to either the intervention or control arms of the trial. Those in the intervention condition were asked to participate in at least 60 minutes of formal volunteering per week for six months. Per-protocol analyses were conducted comparing psychosocial outcomes for those who complied with the intervention condition $(n=73)$ to outcomes for those who complied with the control condition $(n=112)$. Those who complied with the intervention condition demonstrated significant improvements in life satisfaction, purpose in life, and personal growth scores over a 12-month period relative to those in the control condition who did no volunteering. Findings provide evidence of a causal relationship between commencing volunteering and improvements in psychosocial health among older adults and indicate that encouraging participation in this activity could constitute an effective healthy aging intervention.
\end{abstract}

Keywords: Older adults; Volunteering; Randomized controlled trial; Psychosocial health. 


\section{Longitudinal associations between formal volunteering and well-being among retired older people: Follow-up results from a randomized controlled trial}

Populations around the world are aging rapidly, prompting a growing emphasis on healthy aging to ensure increased longevity is accompanied by functional ability and psychological well-being (World Health Organization, 2015). According to the Activity Theory of Aging (Lemon et al., 1972), healthy aging is more likely to occur when older adults maintain their engagement in social and productive activities because they (i) protect older adults from the greater number of role losses that characterize older adulthood and (ii) increase life satisfaction. As a means by which older adults can continue contributing to the community and interacting with others, formal volunteering represents an activity that could be prescribed by health professionals as an intervention to foster healthy aging (Pettigrew et al., 2019).

Cross-sectional and longitudinal research has found older adults' participation in volunteering to be associated with numerous favorable physical and psychosocial health outcomes, including higher cognitive functioning; higher self-rated health; reduced mortality; lower prevalence of hypertension; higher levels of life satisfaction; higher self-esteem, personal growth, and purpose in life; lower rates of depression; greater social connectedness and social support; and reduced loneliness (Anderson et al., 2014; Burr et al., 2015; Carr et al., 2018; Cho et al., 2018; Greenfield \& Marks, 2004; Han \& Hong, 2013; Heo et al., 2017; Jenkinson et al., 2013; Lum \& Lightfoot, 2005; Parkinson et al., 2010; Pettigrew \& Roberts, 2008; Pilkington et al., 2012; Proulx et al., 2018; Tomioka et al., 2017; Wahrendorf et al., 2008). However, a major limitation of this research is the inability to determine whether the observed health differences between volunteers and non-volunteers are a result of their engagement in volunteering or the 
tendency for healthier older adults to be more likely to volunteer in the first place (Jenkinson et al., 2013; Pettigrew et al., 2019). Results from quasi-experimental studies that have attempted to address this limitation indicate that volunteering is associated with (i) fewer depressive symptoms and functional limitations and (ii) greater engagement in physical activity (Hong \& Morrow-Howell, 2010; Tan et al., 2009). However, given the lack of randomization, the potential for confounding bias remains.

Randomized controlled trials (RCTs) are best positioned to definitively establish causality, however few appear to have been conducted to investigate the benefits of commencing volunteering for older people, and results have been mixed (Carlson et al., 2008; Fried et al., 2004; Jiang et al., 2020; Pettigrew et al., 2019; Rook \& Sorkin, 2003; Tan et al., 2006). In a trial that focused on the psychosocial outcomes of volunteering (Rook \& Sorkin, 2003), follow-up assessments conducted one and two years after baseline indicated that older adults randomly assigned to volunteer for a child inpatient hospital visitation program for which they had expressed interest were more likely than those in the comparison non-volunteer groups to form new social ties. However, psychological health (e.g., self-esteem, depression) did not differ between those assigned to participate in this program and those who were not.

In a similar trial that focused on the physical, cognitive, and social outcomes of volunteering (Carlson et al., 2008; Fried et al., 2004; Tan et al., 2006; Varma et al., 2016), older adults who had expressed an interest in volunteering with children were recruited to participate in a school-based intergenerational program (known as Experience Corps) that involved supporting children's literacy development and teaching problem solving and conflict resolution skills. At 4-8 months follow-up, those who participated in the program were more likely than those in the waitlist control group to have favorable physical health outcomes (e.g., feeling 
stronger and smaller declines in walking speed). A significant group difference was also observed in the number of people participants felt they could turn to for help. Changes in psychological health were not assessed.

While these two trials were important in establishing volunteering as a causal predictor of favorable health outcomes, they were limited in several ways. First, most of the older adults recruited into these studies had a pre-existing interest in engaging in child-related volunteering activities and were therefore unlikely to be representative of the broader population of older adults. Second, as the volunteer activities assessed were limited to interacting with children, the potential outcomes of participation in a wider range of volunteering activities were not examined. Third, the amount of volunteering undertaken in each of these trials was substantial, with participants in the child inpatient hospital visitation program volunteering for 20 hours per week and those in the intergenerational program volunteering at least 15 hours per week. The time-intensive nature of the volunteering is likely to have attracted older adults who were especially motivated to participate at this level and capable of doing so.

Two recent RCTs have addressed many of these limitations. The first, conducted by the present author team, involved older adults with no known interest in formal volunteering (blinded for review). Recruiting a broader profile of participants addressed some of the limitations associated with the prior studies' sole focus on older people with a pre-stated intention to undertake volunteering. In addition, those in the intervention condition were able to choose their own volunteering activities, resulting in a wider range of volunteering roles being undertaken compared to those of the previous trials. Finally, to better reflect the reality of older people's volunteering and the competing demands on their time, those in the volunteering 
condition were only required to volunteer for a minimum of 1 hour per week over a 6-month period.

While some differences in physical health outcomes emerged in our RCT between those assigned to the intervention and control conditions at 6-month follow-up (blinded for review), the psychosocial benefits found in previous research were not observed (i.e., higher levels of life satisfaction; higher self-esteem, personal growth, and purpose in life; lower rates of depression; greater social connectedness and social support: Carr et al., 2018; Cho et al., 2018; Greenfield \& Marks, 2004; Han \& Hong, 2013; Heo et al., 2017; Lum \& Lightfoot, 2005; Pilkington et al., 2012; Wahrendorf et al., 2008). These results were partially supported by the second RCT, in which older adults prompted to increase their engagement in voluntary work did not report increased self-efficacy, perceived autonomy, or purpose in life, but did report fewer depressive symptoms at 6-month follow-up (Jiang et al., 2020). Given it has been suggested that the benefits of volunteering are most likely to emerge "in the medium to long term, when social networks and attitudes towards life have had the chance to change" (Russell et al., 2019, p. 119), the 6month follow-up period adopted by both these RCTs may have been too short to enable significant change to occur across many of the assessed psychosocial variables.

The present study aimed to extend our previous research by examining 12-month followup data to determine whether favorable psychosocial outcomes became evident over a longer time period. The outcomes assessed included depressive symptoms, psychological well-being, self-esteem, self-efficacy, purpose in life, personal growth, life satisfaction, and social connectedness.

\section{Method}




\section{Design and procedure}

This RCT was registered with the Australian and New Zealand Clinical Trial Registry (blinded for review) and approval to conduct the research was received from a university Human

Research Ethics Committee. The data reported on in the present study were collected via selfadministered surveys at baseline (Time 1: T1) and at 6-month (Time 2: T2) and 12-month (Time 3: T3) follow-up time points.

A parallel-group design was adopted in which all eligible participants $(n=559)$ were allocated to either the intervention $(n=280)$ or control $(n=279)$ condition based on a computer-generated script using a simple randomization procedure. Those in the intervention condition were asked to undertake a minimum of 60 minutes of formal volunteering per week between T1 and T2. The 60-minute threshold was chosen because prior research has identified a curvilinear relationship between the number of hours spent volunteering and favorable psychological outcomes, with participation in approximately 100 hours of volunteering per year (up to 2 hours per week) considered optimal (Morrow-Howell et al., 2003). Those assigned to the control condition were not asked to volunteer between $\mathrm{T} 1$ and $\mathrm{T} 2$, but given the potential benefits of participation in this activity, for ethical reasons they were not advised that they needed to refrain from volunteering.

Participants in both the intervention and control conditions were informed of the requirements of the study by a member of the study team over the phone and were subsequently given an information sheet. Participants in both conditions were blinded to the purpose of the study (i.e., participants in the control condition were not told about the parallel intervention condition 
involving volunteering, and those in the intervention condition were not told about the control condition that did not involve volunteering).

\section{Recruitment and participants}

Various methods were used to recruit community-dwelling older Australians into the trial. As described elsewhere (blinded for review), these included radio advertising and the placement of notices in newspapers, retirement villages, and the offices of relevant government authorities. All advertising materials indicated the study was examining the health and well-being of older Australians. As per the study protocol (see blinded for review), eligibility criteria were being aged $60+$ years, no engagement in formal volunteering during the previous 12 months, and being retired. Those in paid employment were ineligible because their participation in the workforce was likely to provide psychosocial benefits (Maimaris et al., 2010), potentially confounding the study outcomes.

The demographic profiles of the sample at T1 and T3 are presented in the online supplementary material (Table S1). At T1, 445 Australian older adults participated in the study. All provided informed written consent. Participants ranged in age from 60 to 95 years $(M=$ 70.39 years, $S D=6.07$ ), and $56 \%$ of the sample was female. By T3, 244 participants remained in the study (range 61-96 years, $M=71.44$ years, $S D=6.09,55 \%$ female, $41 \%$ intervention condition), representing an attrition rate of $45 \%$. This rate of attrition is consistent with other longitudinal research involving interventions with older adults (e.g., Busetto et al., 2009; Jancey et al., 2007; Spek et al., 2008). A logistic regression (results of which are presented in the online supplementary material: Table S2) revealed that attrition rates were equal for gender, age, socioeconomic status (SES), and condition allocation, but not health status; those in poorer 
health were more likely than those in better health to withdraw from the study. A CONSORT flow diagram of participants' progress through the phases of the trial is presented in Figure 1. See blinded for review for further information regarding adherence to CONSORT guidelines.

\author{
[Insert Figure 1 about here]
}

\title{
Measures
}

Psychosocial outcomes. The following measures were used at all three time points to assess various aspects of psychosocial health. Depressive symptoms were assessed with the 20-item Center for Epidemiological Studies Depression Scale (Radloff, 1977). Items such as "I felt depressed" were rated on a scale of 1 (Rarely or none of the time) to 4 (Most or all of the time). Cronbach's alphas for scores on this scale at T1, T2, and T3 were $0.87,0.87$, and 0.88 respectively. Psychological well-being was assessed with the 14-item Warwick-Edinburgh Mental Well-Being Scale (Tennant et al., 2007). Responses to items (e.g., I've been feeling relaxed) were made on a scale of 1 (None of the time) to 5 (All of the time). Cronbach's alphas of 0.92, 0.93, and 0.93 were obtained at T1, T2, and T3 respectively. The 10-item Rosenberg SelfEsteem Scale (Rosenberg, 1965) was used to measure self-esteem, with items such as "I feel that I'm a person of worth, at least on an equal plane with others" rated on a scale of 0 (Strongly disagree) to 3 (Strongly agree). Cronbach's alphas for scores on this scale at T1, T2, and T3 were $0.81,0.89$, and 0.88 respectively.

Self-efficacy was assessed using the General Self-Efficacy Scale (Schwarzer \& Jerusalem, 1995). Responses to items such as "No matter what comes my way, I'm usually able to handle it" were made on a scale of 1 (Not at all true) to 4 (Exactly true), with Cronbach's 
alphas of $0.89,0.91$, and 0.91 obtained at T1, T2, and T3 respectively. Purpose in Life and Personal Growth were assessed using the relevant subscales of Ryff's Psychological Well-Being Scales (Ryff, 1989). Responses to items such as "I have a sense of direction and purpose in life" and "I am the kind of person who likes to give new things a try" were made on a scale of 1 (Strongly disagree) to 5 (Strongly agree). Cronbach's alphas of 0.90, 0.88, and 0.90 were obtained at T1, T2, and T3 respectively for Purpose in Life. Cronbach's alphas of 0.86, 0.86, and 0.88 were obtained at T1, T2, and T3 respectively for Personal Growth. Life satisfaction was assessed using a single-item scale adapted from Van Willigen (2000): “All things considered, how satisfied are you with your life as a whole these days?" (Response options: $1=$ Very good to $5=$ Very bad, reverse-scored). Finally, social connectedness was measured using the 24-item Social Provisions Scale (Cutrona \& Russell, 1987). Items (e.g., There is someone I could talk to about important decisions in my life) were rated on a scale that ranged from 1 (Strongly disagree) to 4 (Strongly agree). Cronbach's alphas for scores on this scale at T1, T2, and T3 were $0.91,0.92$, and 0.94 respectively.

Volunteering. At T2 and T3, participants reported whether they had engaged in formal volunteering in the previous 6 months (yes/no response options). Those responding in the affirmative were asked to report the number of organizations for which they had volunteered and the average number of hours per week they engaged in volunteering. Responses to these questions at T2 were used to determine compliance with the conditions of the RCT. Those in the intervention condition who responded 'yes' to engaging in volunteering in the previous 6 months, had volunteered for at least one organization, and had volunteered for a minimum of 60 minutes per week were deemed compliant, as were those in the control condition who responded 
'no' to engaging in volunteering. By contrast, those in the intervention condition who responded 'no' to engaging in volunteering in the previous 6 months, had not volunteered for an organization, or had volunteered for less than 60 minutes per week were deemed non-compliant, as were those in the control condition who responded 'yes' to volunteering. Responses at T3 were used to generate descriptive data on the proportion of participants in the intervention condition who chose to continue volunteering beyond the prescribed intervention period.

Socio-demographics. Participants were asked to report on various socio-demographic characteristics (e.g., gender, age, highest level of education, postcode) at all three time points. Postcodes were used to calculate the SES of the area in which participants resided (as per the Australian Bureau of Statistics' Socioeconomic Index for Areas: Australian Bureau of Statistics, 2018).

\section{Analysis}

As results from the 6-month follow-up have been reported elsewhere (blinded for review), analyses were conducted comparing baseline to 12-month follow-up data only. A per-protocol approach was adopted such that participants who provided $\mathrm{T} 1$ data but did not complete the $\mathrm{T} 2$ or T3 assessments were excluded from analyses. Comparisons were made between those who complied with the intervention condition (i.e., met the minimum 60 minutes per week requirement for volunteering between $\mathrm{T} 1$ and $\mathrm{T} 2$ ) and those who complied with the control condition (i.e., no volunteering between T1 and T2). Sensitivity analyses were also conducted in the form of pragmatic analyses. These compared outcomes for those participants who engaged in 
formal volunteering to those of participants who did not engage in volunteering, regardless of assigned condition.

Paired samples $t$-tests were used to assess changes on the psychosocial outcome variables listed above between T1 and T3 within groups. Hierarchical linear regression analyses were conducted to compare differences between groups. The T1 score of the psychosocial variable under investigation was entered in Step 1 of the analysis. As a gender imbalance was observed by condition assignment owing to the simple rather than stratified randomization procedure adopted, gender was also entered in Step 1 as a control variable (imbalances were not observed for age, SES, or objective health status). The group variable was entered in Step 2. As only 244 participants remained in the sample at T3, bootstrapping ( $n=1000$ replications $)$ was used in estimations to reduce the effect of any excess variability (as per Nevitt \& Hancock, 2001). All analyses were conducted in SPSS.

Among those participants compliant with the volunteering $(n=106)$ and control $(n=$ 178) conditions at $\mathrm{T} 2, n=73$ and $n=112$ participants respectively remained in the sample at $\mathrm{T} 3$. The demographic profile of T3 participants stratified by condition is provided in the online supplementary material (Table S3). Owing to non-response on the items assessing volunteering by some participants, engagement in volunteering between T2 and T3 was only able to be assessed for 160 of these 185 participants. Of these, 41 (66\%) of those who had been compliant with the volunteering condition at $\mathrm{T} 2$ continued their engagement in volunteering between $\mathrm{T} 2$ and T3, while 88 (90\%) of participants compliant with the control condition at T2 remained nonvolunteers. Given these small sample sizes, analyses of the impact of continued volunteering between T2 and T3 were not feasible. 


\section{Results}

\section{Outcomes by allocated condition: per-protocol analyses}

Scores on each of the psychosocial variables under investigation at T1 and T3 stratified by group are presented in Table 1. Despite scores on the outcome variables being moderate to high at T1, significant improvements over time were observed among those in the intervention arm who complied with the study requirements and engaged in volunteering on the variables of life satisfaction $(p<.001)$, social connectedness $(p<.001)$, purpose in life $(p=.001)$, self-esteem $(p$ $=.014)$, and personal growth $(p=.028)$. Improvements over time for two of the outcomes were also observed among those in the control arm who did not engage in volunteering: self-esteem $(p$ $=.001)$ and social connectedness $(p=.008)$.

\section{[Insert Table 1 about here]}

Step 2 results from the per-protocol hierarchical regression analyses are presented in Table 2 (Step 1 results are shown in Table S4 in the online supplementary material). Three significant between-group differences emerged: those in the intervention arm who complied with the study requirements and engaged in volunteering between $\mathrm{T} 1$ and $\mathrm{T} 2$ demonstrated significant improvements in purpose in life $(p=.016)$, personal growth $(p=.020)$, and life satisfaction $(p=$ .020) scores by $\mathrm{T} 3$ (while controlling for $\mathrm{T} 1$ scores and gender) relative to those in the control arm who did not engage in volunteering.

[Insert Table 2 about here] 


\section{Outcomes by actual volunteering status: pragmatic analyses}

Table 3 presents T1 and T3 scores stratified by actual volunteering status (i.e., irrespective of allocated condition). Significant improvements were observed on all variables among those who volunteered between T1 and T2. Among those who did not volunteer, a significant improvement was observed for the variables of self-esteem and social connectedness only (see Table 3 ). One significant between-group difference emerged: those who engaged in volunteering between T1 and T2 demonstrated significant improvements in personal growth $(p=.031)$ scores at T3 (while controlling for T1) relative to those who did not engage in volunteering.

\section{[Insert Table 3 about here]}

\section{Discussion}

This study examined 12-month follow-up data as part of an RCT examining the effects of commencing volunteering on the well-being of older adult non-volunteers (blinded for review). Per-protocol analyses indicated that those in the intervention arm who complied with the study requirements and engaged in volunteering reported significant improvements on the variables of life satisfaction, purpose in life, and personal growth compared to those in the control arm who did not engage in volunteering. Pragmatic analyses indicated that those who volunteered reported significant improvements in personal growth compared to those who did not volunteer, regardless of the condition to which they had been allocated. Outcomes on these psychological variables were not found to differ significantly between groups at the 6-month follow-up (blinded for review), suggesting the impact of volunteering on these outcomes may take some time to manifest and that benefits are more likely to be observed in the medium to long term. 
Given results at 6-month follow-up were trending in the expected direction, it is possible the benefits of volunteering began to emerge in the short term but were not at the level required to reach statistical significance.

These results support the findings of previous cross-sectional and longitudinal research comparing older adult volunteers and non-volunteers (e.g., Cho et al., 2018; Greenfield \& Marks, 2004; Heo et al., 2017; Van Willigen, 2000) by providing evidence of a causal relationship between engagement in volunteering and the variables of life satisfaction, purpose in life, and personal growth. The significant improvements in these outcomes observed among those in the intervention condition compared to those in the control condition also provide support for research that has suggested engagement in volunteering may protect older adults from the reductions in purpose in life and personal growth that can accompany aging due to the loss of major roles such as workforce participation (Greenfield \& Marks, 2004; Heo et al., 2017), thereby facilitating healthy aging (Lemon et al., 1972).

Volunteering was found to have no significant effect on psychological well-being, depression, self-efficacy, self-esteem, or social connectedness. This outcome differs from prior longitudinal and cross-sectional research that found an association between volunteering and these factors (Han \& Hong, 2013; Haski-Leventhal, 2009; Li \& Ferraro, 2005; Morrow-Howell et al., 2003; Musick \& Wilson, 2003). In terms of the results of prior RCTs, the non-significant findings for self-esteem and depression observed in the present study are consistent with outcomes from Rook and Sorkin (2003), but the finding related to depression is inconsistent with the results of Jiang et al. (2020). Further research exploring these mixed results is warranted.

\section{Implications}


The results of the present study have several implications. First, they indicate that volunteering can have important health benefits, providing support for the suggestion that engagement in social and/or productive activities in general has the potential to promote healthy aging (Lemon et al., 1972; Rowe \& Kahn, 1997), and that the 'prescription' of volunteering in particular could constitute an effective healthy aging intervention (Pettigrew et al., 2019). However, it appears that psychological benefits may take some time to manifest, highlighting the need to ensure that older recruits are informed of the likely time periods for these personal benefits to accrue. For example, medical practitioners could recommend volunteering to their patients and explain the time frames over which different kinds of outcomes could be expected. The results of the present RCT suggest that improvements in physical outcomes may occur first (blinded for review), followed later by psychological benefits. Such an approach has the potential to enhance volunteer retention, thereby improving outcomes for individuals and society. It is encouraging that of those participants in the intervention condition who complied with the study requirements and engaged in volunteering between $\mathrm{T} 1$ and $\mathrm{T} 2$, two-thirds chose to continue volunteering after the 6-month trial period was complete and were still volunteering at T3.

Second, the outcomes of this study suggest changes that could be made to the design of future RCTs examining the psychosocial benefits of volunteering. Specifically, it seems prudent to ensure these RCTs are designed to incorporate a follow-up period of at least 12 months to enable detection of changes that take some time to manifest at significant levels. Finally, the loss of a substantial minority of participants to follow-up highlights the importance of designing RCTs in such a way that minimizes attrition. Participants' reasons for withdrawing from the present study were not collected systematically; however, anecdotal reports from those who did provide 
feedback indicated competing commitments and the emergence or exacerbation of health problems were primary reasons. In terms of the former, many of those who provided reasons for their withdrawal reported that they did not have time to participate given their other responsibilities and lifestyle choices, which included (i) providing care to their grandchildren and ill spouses and relatives; (ii) attending meetings or programs of groups, clubs, and organizations to which they belonged (e.g., Probus, University of the Third Age, church groups); and (iii) engaging in spontaneous travel. These tentative findings suggest that researchers designing volunteering RCTs and health practitioners recommending volunteering to patients should be mindful of relevant barriers and assist older people to select activities that can accommodate health conditions, other responsibilities, and busy schedules.

\section{Limitations}

The present study had several limitations. First, as noted above, a substantial minority of participants was lost to follow-up. Although the observed attrition rate is consistent with prior longitudinal research involving interventions with older adults (e.g., Busetto et al., 2009; Jancey et al., 2007; Spek et al., 2008), the sample sizes by condition were too small to permit an assessment of the extent to which continued volunteering between T2 and T3 impacted on the observed outcomes. Second, although attrition rates were equal for gender, age, SES, and condition allocation, participants lost to follow-up had poorer health and may have differed from those who remained at $\mathrm{T} 3$ on factors that were not measured in the present study. Third, baseline scores on the outcome variables were high. This suggests the sample was relatively psychologically healthy, reducing the generalizability of the results to older people in general. In addition, high baseline scores are likely to have made it difficult to observe significant 
improvements over time. Despite this, some statistically significant improvements in psychological health were observed, attesting to the robustness of the findings.

Fourth, the potential impact of the type of volunteering in which participants engaged could not be explored as there was substantial variation in the volunteering roles adopted across the sample, resulting in a lack of statistical power. Research conducted in a larger sample is needed to examine the effect of volunteering type on psychosocial well-being. Finally, the recruitment procedures adopted for the study required participants to self-select, potentially resulting in bias. In addition, all the participants in this follow-up study were volunteers to the extent that they opted to participate, and continue participating, in the study. Research is needed to explore the outcomes associated with commencing volunteering among the broader population of older people, especially among those who are less likely to volunteer for an extended period of time.

\section{Conclusion}

Volunteering commencement was found to be associated with significant increases in older adults' life satisfaction, purpose in life, and personal growth over a 12-month period. These findings provide causal support for the benefits of volunteering among older adults and indicate that encouraging participation in this activity could constitute an effective healthy aging intervention. 


\section{References}

Anderson, N. D., Damianakis, T., Kröger, E., Wagner, L. M., Dawson, D. R., Binns, M. A., Bernstein, S., Caspi, E., \& Cook, S. L. (2014). The benefits associated with volunteering among seniors: A critical review and recommendations for future research. Psychological Bulletin, 140(6), 1505-1533. https://doi.org/10.1037/a0037610

Australian Bureau of Statistics. (2018). Census of Population and Housing: Socio-Economic Indexes for Areas (SEIFA), Australia, 2016 (Cat. no. 2033.0.55.001). Canberra: ABS

Burr, J. A., Han, S. H., \& Tavares, J. L. (2015). Volunteering and cardiovascular disease risk: Does helping others get “under the skin?”. The Gerontologist, 56(5), 937-947. https://doi.org/10.1093/geront/gnv032

Busetto, L., Mazza, M., Salvalaio, S., De Stefano, F., Marangon, M., Calò, E., Sampietro, S., \& Enzi, G. (2009). Obesity treatment in elderly outpatients: predictors of efficacy and dropout. Eating and Weight Disorders - Studies on Anorexia, Bulimia and Obesity, 14, e56e65. https://doi.org/https://doi.org/10.1007/BF03327801

Carlson, M. C., Saczynski, J. S., Rebok, G. W., Seeman, T., Glass, T. A., McGill, S., Tielsch, J., Frick, K. D., Hill, J., \& Fried, L. P. (2008). Exploring the effects of an "everyday" activity program on executive function and memory in older adults: Experience Corps®. The Gerontologist, 48, 793-801. https://doi.org/10.1093/geront/48.6.793

Carr, D. C., Kail, B. L., Matz-Costa, C., \& Shavit, Y. Z. (2018). Does becoming a volunteer attenuate loneliness among recently widowed older adults? The Journals of Gerontology Series B: Psychological Sciences and Social Sciences, 73(3), 501-510. https://doi.org/10.1093/geronb/gbx092

Cho, D., Post, J., \& Kim, S. K. (2018). Comparison of passive and active leisure activities and life satisfaction with aging. Geriatrics \& Gerontology International, 18(3), 380-386. https://doi.org/10.1111/ggi.13188

Cutrona, C. E., \& Russell, D. W. (1987). The provisions of social relationships and adaptation to stress. Advances in Personal Relationships, 1(1), 37-67.

Fried, L. P., Carlson, M. C., Freedman, M., Frick, K. D., Glass, T. A., Hill, J., McGill, S., Rebok, G. W., Seeman, T., Tielsch, J., Wasik, B. A., \& Zeger, S. (2004). A social model for health promotion for an aging population: Initial evidence on the Experience Corps model. Journal of Urban Health, 81(1), 64-78. https://doi.org/10.1093/jurban/jth094

Greenfield, E. A., \& Marks, N. F. (2004). Formal volunteering as a protective factor for older adults' psychological well-being. The Journals of Gerontology Series B: Psychological Sciences and Social Sciences, 59(5), S258-S264. https://doi.org/10.1093/geronb/59.5.S258

Han, C. K., \& Hong, S. I. (2013). Trajectories of volunteering and self-esteem in later life: Does wealth matter? Research on Aging, 35(5), 571-590. https://doi.org/10.1177/0164027512449472

Haski-Leventhal, D. (2009). Elderly volunteering and well-being: A cross-European comparison based on SHARE data. VOLUNTAS: International Journal of Voluntary and Nonprofit Organizations, 20(4), 388-404. https://doi.org/10.1007/s11266-009-9096-X

Heo, J., Chun, S., Kim, B., Ryu, J., \& Lee, Y. (2017). Leisure activities, optimism, and personal growth among the young-old, old-old, and oldest-old. Educational Gerontology, 43(6), 289-299. https://doi.org/10.1080/03601277.2017.1289457 
Hong, S. I., \& Morrow-Howell, N. (2010). Health outcomes of Experience Corps: A highcommitment volunteer program. Social Science \& Medicine. Part A: Medical Sociology, 71, 414-420. https://doi.org/10.1016/j.socscimed.2010.04.009

Jancey, J., Lee, A., Howat, P., Clarke, A., Wang, K., \& Shilton, T. (2007). Reducing attrition in physical activity programs for older adults. Journal of Aging and Physical Activity, 15, 152-165.

Jenkinson, C. E., Dickens, A. P., Jones, K., Thompson-Coon, J., Taylor, R. S., Rogers, M., Bambra, C. L., Lang, I., \& Richards, S. H. (2013). Is volunteering a public health intervention? A systematic review and meta-analysis of the health and survival of volunteers. BMC Public Health, 13. https://doi.org/10.1186/1471-2458-13-773

Jiang, D., Warner, L. M., Chong, A. M. L., Li, T., Wolff, J. K., \& Chou, K. L. (2020). Benefits of volunteering on psychological well-being in older adulthood: Evidence from a randomized controlled trial. Aging \& Mental Health. https://doi.org/10.1080/13607863.2020.1711862

Lemon, B. W., Bengtson, V. L., \& Peterson, J. A. (1972). An exploration of the activity theory of aging: Activity types and life satisfaction among in-movers to a retirement community. Journal of Gerontology, 27(4), 511-523. https://doi.org/10.1093/geronj/27.4.511

Li, Y., \& Ferraro, K. F. (2005). Volunteering and depression in later life: Social benefit or selection processes? Journal of Health and Social Behavior, 46(1), 68-84. https://doi.org/10.1177/002214650504600106

Lum, T. Y., \& Lightfoot, E. (2005). The effects of volunteering on the physical and mental health of older people. Research on Aging, 27(1), 31-55. https://doi.org/10.1177/0164027504271349

Maimaris, W., Hogan, H., \& Lock, K. (2010). The impact of working beyond traditional retirement ages on mental health: implications for public health and welfare policy. Public Health Reviews, 32(2), 532-548. https://doi.org/https://doi.org/10.1007/BF03391615

Morrow-Howell, N., Hinterlong, J., Rozario, P. A., \& Tang, F. (2003). Effects of volunteering on the well-being of older adults. The Journals of Gerontology Series B: Psychological Sciences and Social Sciences, 58(3), S137-S145. https://doi.org/10.1093/geronb/58.3.S137

Musick, M. A., \& Wilson, J. (2003). Volunteering and depression: The role of psychological and social resources in different age groups. Social Science and Medicine, 56(2), 259-269. https://doi.org/10.1016/S0277-9536(02)00025-4

Nevitt, J., \& Hancock, G. R. (2001). Performance of bootstrapping approaches to model test statistics and parameter standard error estimation in structural equation modeling. Structural Equation Modeling, 8(3), 353-377. https://doi.org/10.1207/S15328007SEM0803_2

Parkinson, L., Warburton, J., Sibbritt, D., \& Byles, J. (2010). Volunteering and older women: Psychosocial and health predictors of participation. Aging \& Mental Health, 14(8), 917927. https://doi.org/10.1080/13607861003801045

Pettigrew, S., Jongenelis, M. I., Jackson, B., Warburton, J., \& Newton, R. U. (2019). A randomized controlled trial and pragmatic analysis of the effects of volunteering on the health and well-being of older people. Aging Clinical and Experimental Research. https://doi.org/10.1007/s40520-019-01241-3 
Pettigrew, S., \& Roberts, M. (2008). Addressing loneliness in later life. Aging and Mental Health, 12(3), 302-309. https://doi.org/https://doi.org/10.1080/13607860802121084

Pilkington, P. D., Windsor, T. D., \& Crisp, D. A. (2012). Volunteering and subjective well-being in midlife and older adults: The role of supportive social networks. The Journals of Gerontology Series B: Psychological Sciences and Social Sciences, 67(2), 249-260. https://doi.org/10.1093/geronb/gbr154

Proulx, C. M., Curl, A. L., \& Ermer, A. E. (2018). Longitudinal associations between formal volunteering and cognitive functioning. The Journals of Gerontology Series B: Psychological Sciences and Social Sciences, 73(3), 522-531. https://doi.org/10.1093/geronb/gbx110

Radloff, L. (1977). The CES-D Scale: A self-report depression scale for research in the general population. Applied Psychological Measurement, 1(3), 385-401. https://doi.org/10.1177/014662167700100306

Rook, K. S., \& Sorkin, D. H. (2003). Fostering social ties through a volunteer role: Implications for older-adults' psychological health. The International Journal of Aging and Human Development, 57(4), 313-337. https://doi.org/10.2190/NBBN-EU3H-4Q1N-UXHR

Rosenberg, M. (1965). Society and the adolescent self-image. Princeton University Press.

Rowe, J. W., \& Kahn, R. L. (1997). Successful aging. The Gerontologist, 37(4), 433-440. https://doi.org/10.1093/geront/37.4.433

Russell, A. R., Nyame-Mensah, A., de Wit, A., \& Handy, F. (2019). Volunteering and wellbeing among ageing adults: A longitudinal analysis. VOLUNTAS: International Journal of Voluntary and Nonprofit Organizations, 30(1), 115-128. https://doi.org/10.1007/s11266$\underline{018-0041-8}$

Ryff, C. D. (1989). Happiness is everything, or is it? Explorations on the meaning of psychological well-being. Journal of Personality and Social Psychology, 57(6), 10691081. https://doi.org/10.1037/0022-3514.57.6.1069

Schwarzer, R., \& Jerusalem, M. (1995). Generalized Self-Efficacy scale. In J. Weinman, S. Wright, \& M. Johnston (Eds.), Measures in health psychology: A user's portfolio (pp. 3537). NFER-Nelson.

Spek, V., Cuijpers, P., Nyklicek, I., Smits, N., Riper, H., Keyzer, J., \& Pop, V. (2008). One-year follow-up results of a randomized controlled clinical trial on internet-based cognitive behavioural therapy for subthreshold depression in people over 50 years. Psychological Medicine, 38(5), 1797-1806. https://doi.org/10.1017/S0033291707000542

Tan, E. J., Rebok, G. W., Yu, Q., Frangakis, C. E., Carlson, M. C., Wang, T., Ricks, M., Tanner, E. K., McGill, S., \& Fried, L. P. (2009). The long-term relationship between highintensity volunteering and physical activity in older African American women. The Journals of Gerontology Series B: Psychological Sciences and Social Sciences, 64(2), 304-311.

Tan, E. J., Xue, Q. L., Li, T., Carlson, M. C., \& Fried, L. P. (2006). Volunteering: A physical activity intervention for older adults - the experience Corps ${ }^{\circledR}$ program in Baltimore. Journal of Urban Health, 83(5), 954-969. https://doi.org/https://doi.org/10.1007/s11524006-9060-7

Tennant, R., Hiller, L., Fishwick, R., Platt, S., Joseph, S., Weich, S., Parkinson, J., Secker, J., \& Stewart-Brown, S. (2007). The Warwick-Edinburgh Mental Well-Being Scale (WEMWBS): Development and UK validation. Health and Quality of Life Outcomes, 5. https://doi.org/10.1186/1477-7525-5-63 
Tomioka, K., Kurumatani, N., \& Hosoi, H. (2017). Association between the frequency and autonomy of social participation and self-rated health. Geriatrics \& Gerontology International, 17(12), 2537-2544. https://doi.org/10.1111/ggi.13074

Van Willigen, M. (2000). Differential benefits of volunteering across the life course. The Journals of Gerontology Series B: Psychological Sciences and Social Sciences, 55(5), S308-S318. https://doi.org/10.1093/geronb/55.5.S308

Varma, V. R., Tan, E. J., Gross, A. L., Harris, G., Romani, W., Fried, L. P., Rebok, G. W., \& Carlson, M. C. (2016). Effect of community volunteering on physical activity: A randomized controlled trial. American Journal of Preventive Medicine, 50(1), 106-110. https://doi.org/10.1016/j.amepre.2015.06.015

Wahrendorf, M., Ribet, C., Zins, M., \& Siegrist, J. (2008). Social productivity and depressive symptoms in early old age-results from the GAZEL study. Aging and Mental Health, 12(3), 310-316. https://doi.org/https://doi.org/10.1080/13607860802120805

World Health Organization. (2015). World report on ageing and health. Geneva: WHO 


\section{Table 1}

Outcomes by Allocated Condition ( $n=185)$ : Per-Protocol Analyses

\begin{tabular}{|c|c|c|c|c|c|c|c|c|c|c|c|c|}
\hline \multirow[t]{3}{*}{ Measures } & \multicolumn{6}{|c|}{ Volunteer condition: compliant $^{\mathrm{a}}(n=73)$} & \multicolumn{6}{|c|}{ Control condition: compliant $^{\mathrm{b}}(n=112)$} \\
\hline & \multicolumn{2}{|c|}{$\mathrm{T} 1$} & \multicolumn{2}{|c|}{ T3 } & \multicolumn{2}{|c|}{$\mathrm{T} 1-\mathrm{T} 3$} & \multicolumn{2}{|c|}{$\mathrm{T} 1$} & \multicolumn{2}{|c|}{ T3 } & \multicolumn{2}{|c|}{ T1-T3 } \\
\hline & $\mathrm{M}$ & $S D$ & $\mathrm{M}$ & $S D$ & $\Delta$ & $d$ & $\mathrm{M}$ & $S D$ & $\mathrm{M}$ & $S D$ & $\Delta$ & $d$ \\
\hline Psychological well-being (range: $14-70$ ) & 56.07 & 7.97 & 57.76 & 7.61 & 1.69 & 0.21 & 54.36 & 7.80 & 55.01 & 8.42 & 0.64 & 0.09 \\
\hline Depression (range: $0-60$ ) & 8.39 & 7.65 & 6.90 & 6.75 & -1.49 & -0.22 & 7.49 & 7.71 & 7.69 & 7.80 & 0.21 & 0.03 \\
\hline Self-efficacy (range: $10-40$ ) & 33.29 & 3.97 & 33.99 & 4.49 & 0.70 & 0.22 & 31.88 & 3.96 & 32.47 & 4.39 & 0.59 & 0.18 \\
\hline Purpose in life (range: $14-70$ ) & 67.13 & 12.66 & 70.84 & 10.81 & $3.70 * *$ & 0.42 & 66.58 & 12.01 & 67.14 & 11.78 & 0.55 & 0.07 \\
\hline Personal growth (range: $14-70$ ) & 70.38 & 10.39 & 73.28 & 9.21 & $2.90 *$ & 0.27 & 68.41 & 9.10 & 68.48 & 9.89 & 0.06 & 0.01 \\
\hline Self-esteem (range: $0-30)$ & 23.55 & 4.90 & 24.97 & 4.34 & $1.42 *$ & 0.31 & 23.68 & 5.47 & 25.08 & 4.57 & $1.41 * *$ & 0.33 \\
\hline Life satisfaction (range: $1-5$ ) & 4.07 & 0.77 & 4.36 & 0.64 & $0.29 * * *$ & 0.42 & 4.08 & 0.79 & 4.14 & 0.75 & 0.06 & 0.07 \\
\hline Social connectedness (range: $24-96$ ) & 79.79 & 10.26 & 82.89 & 10.92 & $3.11 * * *$ & 0.47 & 79.02 & 9.14 & 81.20 & 10.14 & $2.18 * *$ & 0.27 \\
\hline
\end{tabular}

Note. Those who completed their T3 assessment but did not provide data relating to one or more of the outcome variables were treated listwise.

aResponded 'yes' to engaging in volunteering in the previous 6 months, had volunteered for at least one organization, and had volunteered for an average of 60 minutes per week.

'Responded 'no' to engaging in volunteering.

$* p<.05 . * * p<.01 . * * * p<.001$. 
Table 2

Per-Protocol Hierarchical Regression Analyses $(n=185)$

\begin{tabular}{|c|c|c|c|c|c|c|}
\hline Dependent variable & Independent variable & $\mathrm{B}$ & $S E$ & $\beta$ & $p$ & $95 \% \mathrm{CI}$ \\
\hline \multirow[t]{3}{*}{ T3 psychological well-being } & Gender & 0.78 & 1.12 & .05 & .504 & $-1.44,2.93$ \\
\hline & T1 psychological well-being & 0.59 & 0.10 & .57 & $<.001$ & $0.40,0.79$ \\
\hline & Group & 1.58 & 1.11 & .09 & .159 & $-0.56,3.81$ \\
\hline \multirow[t]{3}{*}{ T3 depression } & Gender & -0.88 & 1.03 & -.06 & .403 & $-2.87,1.21$ \\
\hline & T1 depression & 0.53 & 0.10 & .54 & $<.001$ & $0.33,0.72$ \\
\hline & Group & -1.06 & 1.04 & -.07 & .298 & $-3.23,1.02$ \\
\hline \multirow[t]{3}{*}{ T3 self-efficacy } & Gender & 1.01 & 0.46 & .11 & .035 & $0.13,2.01$ \\
\hline & T1 self-efficacy & 0.78 & 0.06 & .70 & $<.001$ & $0.65,0.90$ \\
\hline & Group & 0.22 & 0.49 & -.02 & .647 & $-0.81,1.13$ \\
\hline \multirow[t]{3}{*}{$\mathrm{T} 3$ purpose in life } & Gender & 0.50 & 1.29 & .02 & .695 & $-1.93,3.04$ \\
\hline & $\mathrm{T} 1$ purpose in life & 0.69 & 0.08 & .73 & $<.001$ & $0.54,0.84$ \\
\hline & Group & 3.22 & 1.25 & .14 & .016 & $0.62,5.50$ \\
\hline \multirow{3}{*}{ T3 personal growth } & Gender & 2.04 & 1.22 & .10 & .106 & $-0.28,4.46$ \\
\hline & $\mathrm{T} 1$ personal growth & 0.58 & 0.09 & .56 & $<.001$ & $0.41,0.76$ \\
\hline & Group & 3.26 & 1.28 & .16 & .020 & $0.52,5.80$ \\
\hline \multirow[t]{3}{*}{ T3 self-esteem } & Gender & 0.70 & 0.54 & .08 & .192 & $-0.41,1.79$ \\
\hline & T1 self-esteem & 0.51 & 0.09 & .60 & $<.001$ & $0.35,0.69$ \\
\hline & Group & -0.21 & 0.59 & -.02 & .732 & $-1.37,0.96$ \\
\hline \multirow[t]{3}{*}{ T3 life satisfaction } & Gender & -0.02 & 0.11 & -.01 & .869 & $-0.24,0.21$ \\
\hline & T1 life satisfaction & 0.42 & 0.09 & .46 & $<.001$ & $0.26,0.60$ \\
\hline & Group & 0.23 & 0.10 & .16 & .020 & $0.04,0.42$ \\
\hline \multirow[t]{3}{*}{ T3 social connectedness } & Gender & 1.43 & 1.21 & .07 & .251 & $-0.90,3.82$ \\
\hline & T1 social connectedness & 0.77 & 0.06 & .70 & $<.001$ & $0.65,0.88$ \\
\hline & Group & 0.82 & 1.10 & .04 & .468 & $-1.44,2.96$ \\
\hline
\end{tabular}

Note. Significant group effect shown in bold text. $\beta=$ not bootstrapped. Group: $1=$ compliant with control condition, $2=$ compliant with intervention condition. Gender: $1=$ male, 2 = female. Step 1 of hierarchical regression analysis not presented. 
Table 3

Outcomes by Volunteering Status, Irrespective of Allocated Condition $\left(n=229^{a}\right)$ : Pragmatic Analyses

\begin{tabular}{|c|c|c|c|c|c|c|c|c|c|c|c|c|c|}
\hline \multirow[t]{3}{*}{ Measures } & \multicolumn{6}{|c|}{ Volunteered between T1 and T2 $(n=101)$} & \multicolumn{6}{|c|}{ Did not volunteer between T1 and T2 $(n=128)$} & \multirow[t]{3}{*}{ Regression results } \\
\hline & \multicolumn{2}{|c|}{$\mathrm{T} 1$} & \multicolumn{2}{|c|}{ T3 } & \multicolumn{2}{|c|}{$\mathrm{T} 1-\mathrm{T} 3$} & \multicolumn{2}{|c|}{$\mathrm{T} 1$} & \multicolumn{2}{|c|}{ T3 } & \multicolumn{2}{|c|}{$\mathrm{T} 1-\mathrm{T} 3$} & \\
\hline & $\mathrm{M}$ & $S D$ & $\mathrm{M}$ & $S D$ & $\Delta$ & $d$ & $\mathrm{M}$ & $S D$ & $\mathrm{M}$ & $S D$ & $\Delta$ & $d$ & \\
\hline Psychological well-being & 55.74 & 7.85 & 57.81 & 8.14 & $2.06^{*}$ & 0.26 & 54.67 & 7.64 & 55.34 & 31 & 0.67 & 0.10 & $\begin{array}{r}\mathrm{B}=-1.77, S E=0.95, \beta=-.11, p=.069 \\
95 \% \mathrm{CI}=-3.59,0.17\end{array}$ \\
\hline Depression & 8.11 & 7.19 & 6.42 & 6.50 & $-1.69 *$ & -0.28 & 7.37 & 7.35 & 7.37 & 7.44 & 0.00 & 0.00 & $\begin{array}{r}\mathrm{B}=1.28, S E=0.83, \beta=.09, p=.125, \\
95 \% \mathrm{CI}=-0.34,2.88\end{array}$ \\
\hline Self-efficacy & 33.19 & 3.79 & 34.00 & 4.34 & $0.81 *$ & 0.25 & 32.09 & 4.01 & 32.68 & 4.41 & 0.59 & 0.18 & $\begin{array}{r}\mathrm{B}=-0.38, S E=0.42, \beta=-.04, p=.580, \\
95 \% \mathrm{CI}=-5.11,9.04\end{array}$ \\
\hline Purpose in life & 67.46 & 12.38 & 69.93 & 10.70 & $2.46^{*}$ & 0.27 & 67.08 & 11.65 & 67.87 & 11.52 & 0.79 & 0.10 & $\begin{array}{r}\mathrm{B}=-1.66, S E=1.11, \beta=-.07, p=.152 \\
95 \% \mathrm{CI}=-3.87,0.56\end{array}$ \\
\hline Personal growth & 70.94 & 10.20 & 72.98 & 9.38 & $2.04 *$ & 0.20 & 68.56 & 9.24 & 68.97 & 9.62 & 0.41 & 0.05 & $\begin{array}{r}\mathrm{B}=-\mathbf{2 . 4 4}, S E=1.08, \beta=-.13, p=.031 \\
95 \% \mathrm{CI}=-4.43,-\mathbf{0 . 2 3}\end{array}$ \\
\hline Self-esteem & 23.61 & 4.98 & 25.05 & 4.63 & $1.44 * *$ & 0.31 & 23.92 & 5.31 & 25.25 & 4.43 & $1.33 * * *$ & 0.33 & $\begin{array}{r}\mathrm{B}=0.12, S E=0.53, \beta=.01, p=.822, \\
95 \% \mathrm{CI}=-0.94,1.15\end{array}$ \\
\hline Life satisfaction & 4.03 & 0.81 & 4.26 & 0.74 & $0.22 * *$ & 0.29 & 4.11 & 0.76 & 4.17 & 0.73 & 0.06 & 0.07 & $\begin{array}{r}\mathrm{B}=-0.12, S E=0.09, \beta=-.08, p=.201, \\
95 \% \mathrm{CI}=-0.29,0.07\end{array}$ \\
\hline Social connectedness & 79.25 & 10.57 & 82.40 & 10.50 & $3.15 * * *$ & 0.46 & 79.31 & 9.05 & 81.61 & 9.90 & $2.30 * *$ & 0.29 & $\begin{array}{r}\mathrm{B}=-0.74, S E=0.99, \beta=-.04, p=.451, \\
95 \% \mathrm{CI}=-2.65,1.19\end{array}$ \\
\hline
\end{tabular}

Note. Those who completed their T3 assessment but did not provide data relating to one or more of the outcome variables were treated listwise. Significant group

effect shown in bold text. Gender treated as a covariate. $\beta=$ not bootstrapped.

$* p<.05 . * * p<.01 . * * * p<.001$.

${ }^{a}$ Volunteering status was unable to be determined for 15 participants (excluded from analyses). 


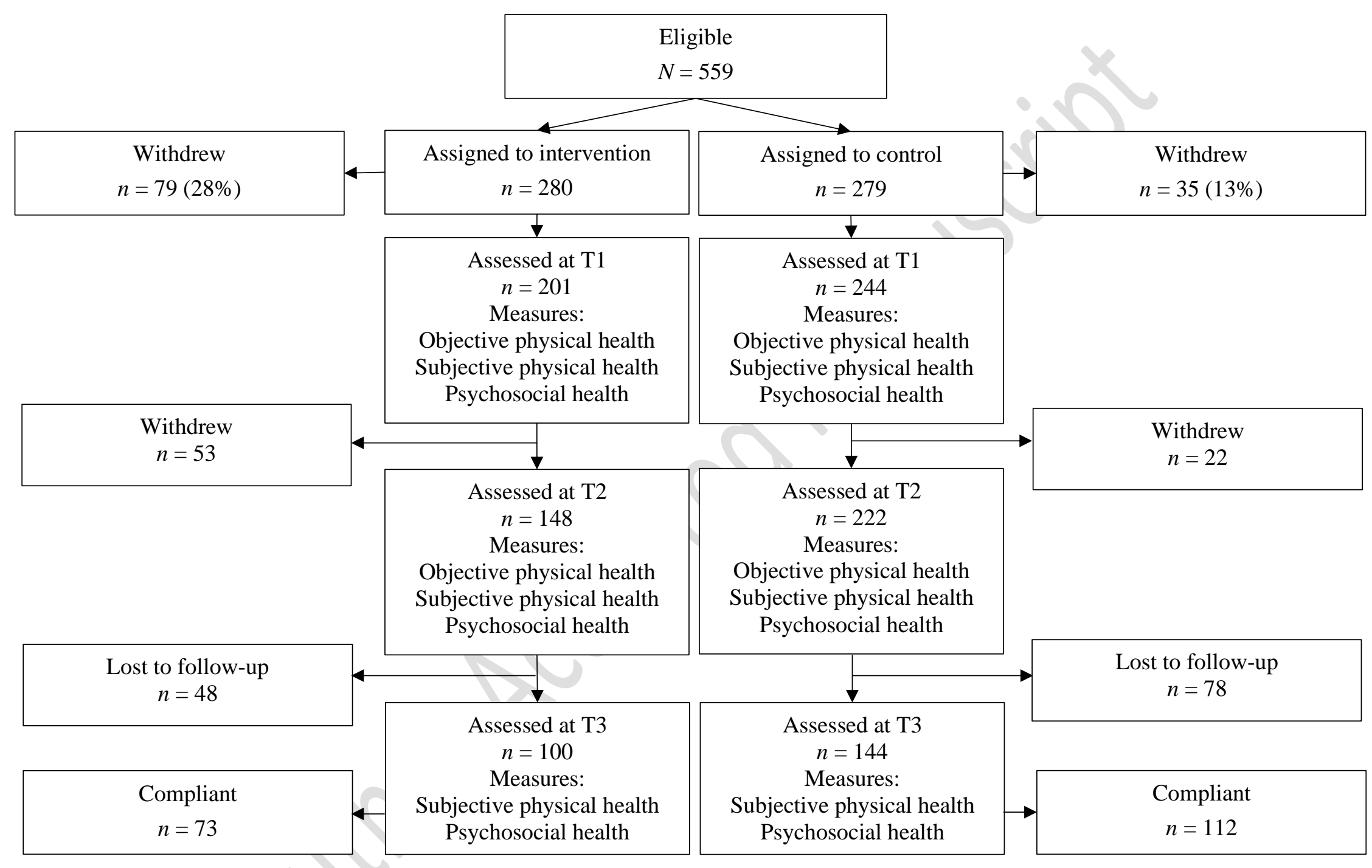




\section{Figure 1}

CONSORT Diagram Depicting Progress Through the Study and the Measures Collected at Each Time Point. 


\section{University Library}

\section{- M I N E R VA \\ A gateway to Melbourne's research publications}

Minerva Access is the Institutional Repository of The University of Melbourne

\section{Author/s:}

Jongenelis, M;Jackson, B;Newton, RU;Pettigrew, S

Title:

Longitudinal associations between formal volunteering and well-being among retired older people: follow-up results from a randomized controlled trial

\section{Date:}

2021-02-04

\section{Citation:}

Jongenelis, M., Jackson, B., Newton, R. U. \& Pettigrew, S. (2021). Longitudinal associations between formal volunteering and well-being among retired older people: follow-up results from a randomized controlled trial. AGING \& MENTAL HEALTH, 26 (2), pp.368-375. https:// doi.org/10.1080/13607863.2021.1884845.

Persistent Link:

http://hdl.handle.net/11343/297104 